\title{
Ranibizumab For Diabetic Macular Edema Refractory To Multiple Prior Treatments
}

Thomas A. Ciulla, M.D. ${ }^{1}$, Rehan M. Hussain, M.D. ${ }^{2}$, Lauren Ciulla ${ }^{1}$, Bethany Sink $^{1}$, Alon Harris, Ph.D. ${ }^{2}$

Corresponding Author:

Thomas A. Ciulla, M.D.

Retina Service

Midwest Eye Institute

200 W. $103^{\text {rd }}$ Street

Indianapolis, IN 46290

Thomasciulla@gmail.com

Phone: 317-817-1822

TC receives contracted research funding from Acucela, Alcon, Ampio, Lpath, Ohr, Ophthotec, Pfizer, Thrombogenics, and Xoma. He is a consultant for Ohr, Stealth, and Thrombogenics. AH receives remuneration from MSD and Alcon for serving as a lecturer and from Merck, Pharmalight, Sucampo, Biolight, Nanoretina, and ONO Pharmaceuticals for serving as a consultant. AH also holds an ownership interest Adom (which all relationships above are pursuant to IU's policy on outside activities). None of the other authors have any financial disclosures.

This is the author's manuscript of the article published in final edited form as:

Ciulla, T. A., Hussain, R. M., Ciulla, L. M., Sink, B., \& Harris, A. (2016). Ranibizumab For Diabetic Macular Edema Refractory To Multiple Prior Treatments. RETINA, 36(7), 1292-1297. http://dx.doi.org/10.1097/IAE.0000000000000876

${ }^{1}$ Retina Service, Midwest Eye Institute, 200 W. 103 ${ }^{\text {rd }}$ St, Indianapolis, IN 46290

2 Department of Ophthalmology, Indiana University School of Medicine, $1160 \mathrm{~W}$. Michigan St, Indianapolis, IN 46202 
Key words: diabetic macular edema, diabetic retinopathy, Lucentis, macular edema, ranibizumab

Summary statement: We reviewed 33 eyes of 22 patients with refractory diabetic macular edema, treated with an average of six $0.3 \mathrm{mg}$ intravitreal ranibizumab injections over 48 weeks. Mean visual acuity and central subfield thickness showed statistically significant linear trends in improvement with respect to days of follow up. 


\section{Abstract \\ Purpose:}

Diabetic macular edema (DME) can be refractory to multiple treatment modalities. Although there have been anecdotal reports of ranibizumab showing efficacy when other modalities provided limited benefit, there has been little published on treatment for refractory DME. This study sought to investigate this observation further.

\section{Methods:}

Retrospective chart review.

\section{Results:}

33 eyes of 22 patients with refractory DME were treated with $0.3 \mathrm{mg}$ intravitreal ranibizumab. This group of eyes received an average of 5.1 prior treatments (macular laser, intravitreal bevacizumab, triamcinolone acetonide, or dexamethasone implant). The mean best corrected visual acuity (BCVA) prior to the initial ranibizumab injection was 20/110 and the mean central subfield thickness (CSFT) was $384 \mu \mathrm{m}$. After 7 visits over an average of 48 weeks, during which an average of 6 ranibizumab injections were administered, the mean VA improved to 20/90 and the mean CSFT improved to $335 \mu \mathrm{m}$. Both CSFT and BCVA improved with number of days of follow up in a statistically significant fashion $(\mathrm{P}<0.01)$. Similarly, both CSFT and VA improved with number of ranibizumab injections in a linear fashion, but this was not statistically significant.

\section{Conclusion}

Ranibizumab can improve DME refractory to prior treatments of laser photocoagulation, intravitreal triamcinolone acetonide, and bevacizumab. 


\section{Introduction}

Diabetic macular edema (DME) can be refractory to multiple treatment modalities. The literature on the treatment of refractory DME is limited. There have been anecdotal reports of intravitreal ranibizumab showing efficacy when other treatment modalities provide limited benefit. This study sought to investigate this observation further through a retrospective chart review in DME patients refractory to multiple prior treatments, who were treated with $0.3 \mathrm{mg}$ intravitreal ranibizumab.

\section{Methods}

This retrospective, uncontrolled chart review studied consecutive patients diagnosed with refractory DME who were treated with their first intravitreal ranibizumab injections from November 2012 through December 2013. This project was reviewed by Indiana University’s IRB and considered exempt. Refractory DME was diagnosed if the patients showed persistent DME of at least 6 months duration despite at least 2 prior treatments, including any combination of macular laser photocoagulation, intravitreal triamcinolone acetonide, intravitreal bevacizumab, or intravitreal dexamethasone implant. Only patients diagnosed with diabetes and using either an oral antihyperglycemic agent or insulin were included.

Exclusion criteria included other causes of macular edema, such as venous occlusion or age-related macular degeneration. Patients were excluded if the baseline best corrected visual acuity (BCVA) was better than 20/40 or the central subfield thickness (CSFT) on spectral domain optical coherence tomography (OCT) was less than 
300 microns. A minimum of 6 months of follow-up was required to be eligible for the study.

Patient charts were reviewed for eligibility, and data were extracted regarding the patient's age, gender, previous interventions, and OCT CSFT. The BCVA, CSFT, treatment dates from the initial visit and follow-up visits were recorded. Snellen visual acuity was converted to $\log$ MAR visual acuity (logMAR VA) for statistically analysis. In cases of missing data points, the last observation was carried forward. Regression analysis was performed on the individual data to determine correlations between the independent variables of number of days follow-up or number of ranibizumab injections and the dependent variables of logMAR VA or CSFT. Mean values for LogMAR VA and CSFT, as well as standard deviations and range, were calculated at each follow up visit. Fluorescein angiography was performed on each patient at their initial presentation to the clinic; eyes with macular ischemia (foveal avascular zone $>1$ disk area) were excluded from the study.

On all visits, response to treatment was evaluated subjectively by Snellen visual acuity with best correction, and objectively by biomicroscopic examination and Zeiss Cirrus spectral domain OCT (Carl Zeiss Meditec, Jena, Germany). In all cases, the use of ranibizumab and its potential risks and benefits were discussed with the patients before signing an informed consent. Ranibizumab $0.3 \mathrm{mg}$ was injected $3.5-4 \mathrm{~mm}$ posterior to the limbus under aseptic conditions. Patients were assessed every 6 weeks on average. Subsequent injections were administered for persistent macular edema on OCT.

\section{Results:}


The study included 33 eyes of 22 patients with refractory DME. The patient demographic and baseline characteristics are summarized in Table 1. Follow up after treatment with ranibizumab ranged from 200 to 622 days; data was analyzed up to the seventh visit at a mean of 338 days, and any missing data was carried forward. In 4 patients the left eye was affected and in 7 the right eye was affected. 11patients had bilateral involvement. All patients had DME for a least 6 months duration prior to undergoing ranibizumab treatment. Prior to treatment with ranibizumab, this group of eyes received an average of 5.6 +/- 2.9 prior treatments (33 eyes received 2-14 treatments including macular laser, intravitreal bevacizumab, triamcinolone acetonide or dexamethasone implant). Specifically, these 33 eyes received an average of $1.7+/-1.4$ macular laser treatments (27 eyes received 1-6 treatments), 0.4 +/- 0.8 intravitreal triamcinolone acetonide (9 eyes received 1-3 treatments), 3.4+/- 1.7 bevacizumab (33 eyes received 1-6 treatments). Both eyes of one patient received 2 prior dexamethasone implants. Macular laser treatment was performed using a modified ETDRS protocol in which all leaking microaneuryms were directly treated to achieve a mild gray-white burn, followed by grid treatment to areas with diffuse leakage, between 500 and 3000 microns from the fovea; $532 \mathrm{~nm}$ laser was set to spot size of 50 microns at 0.05 to $0.1 \mathrm{sec}$. The mean time period between the prior treatment and the initial ranibizumab injection was 167 days (range 29 - 455 days). The previous treatments are summarized in Table 2.

The mean BCVA prior to the initial ranibizumab injection was 20/110 (logMAR $0.52+/-0.38$ ) and the mean CSFT was $384+/-129 \mu \mathrm{m}$. After 7 visits over an average of 48 weeks, during which an average of 6 ranibizumab injections were administered, the 
mean VA improved to 20/90 (logMAR $0.44+/-0.40)$ and the mean CSFT improved to $335+/-164 \mu \mathrm{m}$. These data are summarized in Table 3.

Both CSFT and BCVA improved with number of days of follow up in a statistically significant fashion $(\mathrm{P}<0.01)$. Similarly, both CSFT and BCVA improved with number of ranibizumab injections, but this was not statistically significant. There was a linear trend in improvement of mean CSFT compared to mean number of days of follow-up $\left(\mathrm{R}^{2}=0.51\right)$, mean CSFT compared to mean number of ranibizumab injections $\left(\mathrm{R}^{2}=0.52\right)$, mean logMAR VA compared to mean number days of follow-up $\left(\mathrm{R}^{2}=0.74\right)$, and mean logMAR VA compared to mean number of ranibizumab injections $\left(\mathrm{R}^{2}=0.74\right)$. These data are presented graphically Figure 1.There were no cases of endophthalmitis, retinal detachment, arteriothrombotic events, or death throughout the study period.

\section{Discussion}

Intravitreal anti-vascular endothelial growth factor (anti-VEGF) agents have become first line therapy in diabetic macular edema (DME) patients, as several recent clinical trials suggest that these therapies are more effective than laser photocoagulation for DME. ${ }^{1-5}$ Intravitreal bevacizumab has been used off-label for the treatment DME for a number of years. In 2012, intravitreal ranibizumab was approved by the FDA for DME, based on Genentech’s Phase III trials, RIDE and RISE. ${ }^{6,7}$

More recently, there has been interest in intravitreal corticosteroids, as the dexamethasone implant ${ }^{8}$ and the fluocinolone implant ${ }^{9,10}$ have been approved for the treatment of DME in 2014 and 2015, respectively. There are few large prospective clinical trials comparing anti-VEGF agents to corticosteroids; DRCR protocol I compared 
$0.5 \mathrm{mg}$ ranibizumab to $4 \mathrm{mg}$ intravitreal triamcinolone acetonide; when analysis was confined to the pseudophakic group of patients to control for the effect of cataract formation, the group that received intravitreal triamcinolone acetonide showed similar visual acuity results to the group that received ranibizumab.

In 2014, intravitreal aflibercept was approved for the treatment of DME based on Regeneron's Phase 3 VISTA-DME and VIVID-DME trials. ${ }^{11}$ Even more recently, aflibercept was compared to ranibizumab and bevacizumab for the treatment of DME in the Diabetic Retinopathy Clinical Research Network (DRCR.net) comparative effectiveness study in 660 patients with Diabetic Macular Edema (Protocol T). ${ }^{12}$ In this study, aflibercept demonstrated a significantly greater improvement in mean change in BCVA from baseline at 52 weeks compared to both bevacizumab and ranibizumab injection, in the subgroups with baseline visual acuity of 20/50 or worse, but there was no significant difference in visual acuity between the subgroups with better baseline visual acuity (between 20/32 -20/40). Anatomically, aflibercept and ranibizumab demonstrated a statistically significant greater reduction of CSFT compared to bevacizumab, with values of $-169 \mu \mathrm{m},-147 \mu \mathrm{m}$, and $-101 \mu \mathrm{m}$, respectively. These results held true regardless of initial baseline VA, though there was no statistically significant difference in anatomic efficacy when comparing ranibizumab to aflibercept. These results suggest that either aflibercept or ranibizumab would be of potential benefit for treating persistent DME after trial with bevacizumab.

Despite this growing clinical research data, numerous questions remain. Most clinicians do not follow dosing regimens employed in registration trials, as many will follow an off-label "treat and extend” regimen, which has not been studied in large 
randomized clinical trials. In addition, many clinicians will switch between agents when there is suboptimal response, as DME can be refractory to multiple treatment modalities. The REEF study (prospective nonrandomized trial of 43 patients) found benefit in switching from bevacizumab to three monthly injections of $0.5 \mathrm{mg}$ ranibizumab, with mean additional reduction of $113 \mu \mathrm{m}$ and gain of 6.4 letters by 3 month follow up. As of now, there are no large randomized prospective clinical trials comparing treatment regimens for refractory DME to guide treatment regimens.

This study suggests that Ranibizumab can improve DME refractory to some prior treatments, including laser photocoagulation, intravitreal triamcinolone acetonide, and bevacizumab. Although the improvement in visual acuity and CSFT is modest, these cases can be especially difficult to treat, and consequently these modest improvements can be clinically meaningful, especially in patients with bilateral refractory DME. This study has several limitations, including its retrospective design without a standardized refraction protocol, lack of controls, and lack of long-term follow up. Also, tachyphylaxis may be a confounder, that the benefit sometimes observed from simply switching from one therapy to another may represent a limitation of this study, potentially accounting for the modest benefit observed. In addition, there was a mean wash out period of 167 days (range 29 - 455 days) between prior therapy and initiation of ranibizumab, and it is possible that the absence of recent therapy contributed to the modest improvement in DME. Furthermore, as another limitation of this retrospective study, the average treatment frequency of ranibizumab was less than the recommended monthly treatment frequency; more frequent monthly treatment would have likely shown a more robust improvement in DME after switching to ranibizumab. The mechanism by which 
ranibizumab may exert more complete effect over the other treatments is unclear, but anti-VEGF agents have been shown to have different efficacies in treatment-naïve DME with poor baseline visual acuity, as noted above. Furthermore, given the result of DRCR.net protocol $\mathrm{T}$, it is possible that switching therapy to aflibercept could provide even more benefit in cases of refractory DME. ${ }^{12}$ Bevacizumab, ranibizumab, and aflibercept each have different binding affinities for VEGF, as well as different half-lives in the vitreous, and this could account for some of the differences noted between these agents.

Truly chronic DME may differ even more in pathophysiology from non-chronic DME. A recent study compared the efficacy of a fluocinolone sustained release implant in chronic ( $>$ /= 3 years) versus nonchronic ( $<3$ years) DME in a preplanned subgroup analysis of a large prospective randomized trial. ${ }^{13}$ At month 36, the difference between fluocinolone implant and sham control in the percentage of subjects who gained 15 letters or more was significantly greater in 536 chronic DME subjects (34.0\% vs. sham, 13.4\%; $\mathrm{P}<0.001)$, compared to the 416 subjects with nonchronic DME (22.3\% vs. sham, $27.8 \%$; $\mathrm{P}=0.275$ ). The differences could not be explained by baseline ocular characteristics, changes in anatomic features, or differences in re-treatment or ancillary therapies. The authors speculate that early DME is driven primarily by VEGF, while chronic DME may be driven more by inflammatory cytokines in addition to anatomic changes, and that intravitreal corticosteroids will inhibit the release of these inflammatory cytokines.

Currently, the DRCR Protocol $U$ is exploring the efficacy of combination therapy with $0.3 \mathrm{mg}$ ranibizumab and dexamethasone intravitreal implant compared to continued therapy with $0.3 \mathrm{mg}$ ranibizumab to treat persistent DME. Our results, along with those 
of DRCR Protocol T, suggest the need for large prospective randomized trials to validate that switching from either bevacizumab to ranibizumab or aflibercept is superior to continued bevacizumab treatment in cases of persistent DME. Clearly, further study of refractory DME is warranted, given the visual disability caused.

\section{References}

1. Diabetic Retinopathy Clinical Research Network. Randomized trial evaluating ranibizumab plus prompt or deferred laser or triamcinolone plus prompt laser for diabetic macular edema. Ophthalmology 2010;117:1064-77

2. Diabetic Retinopathy Clinical Research Network. Intravitreal ranibizumab for diabetic macular edema with prompt versus deferred laser treatment: three-year randomized trial results. Ophthalmology 2012;119:2312-8.

3. Do DV, Nguyen QD, Khwaja AA, et al. Ranibizumab for edema of the macula in diabetes study: 3-year outcomes and the need for prolonged frequent treatment. JAMA Ophthalmol 2013;131:139-45.

4. Elman MJ, Ayala A, Bressler NM, et al. Intravitreal Ranibizumab for Diabetic Macular Edema with Prompt versus Deferred Laser Treatment: 5-Year Randomized Trial Results. Ophthalmology 2015;122:375-81

5. Nguyen QD, Shah SM, Khwaja AA, et al. Two-year outcomes of the ranibizumab for edema of the macula in diabetes (READ-2) study. Ophthalmology 2010; 117:2146-51.

6. Brown DM, Nguyen QD, Marcus DM, et al. Long-term outcomes of ranibizumab therapy for diabetic macular edema: the 36-month results from two phase III trials: RISE and RIDE. Ophthalmology 2013;120:2013-22.

7. Nguyen QD, Brown DM, Marcus DM, et al. Ranibizumab for diabetic macular edema: results from 2 phase III randomized trials: RISE and RIDE. Ophthalmology 2012;119:789-801.

8. Boyer DS, Yoon YH, Belfort R Jr, et al. Three-year, randomized, sham-controlled trial of dexamethasone intravitreal implant in patients with diabetic macular edema. Ophthalmology 2014;121:1904-14.

9. Campochiaro PA, Brown DM, Pearson A, et al. Sustained delivery fluocinolone acetonide vitreous inserts provide benefit for at least 3 years in patients with diabetic macular edema. Ophthalmology 2012;119:2125-32.

10. Campochiaro PA, Brown DM, Pearson A, et al. Long-term benefit of sustaineddelivery fluocinolone acetonide vitreous inserts for diabetic macular edema. Ophthalmology 2011;118:626-635.

11. Korobelnik JF, Do DV, Schmidt-Erfurth U, et al. Intravitreal aflibercept for diabetic macular edema. Ophthalmology 2014;121;2247-54.

12. The Diabetic Retinopathy Clinical Research Network. Aflibercept, Bevacizumab, or Ranibizumab for Diabetic Macular Edema. N Engl J Med 2015;372:1193-203. 
13. Cunha-Vaz J, Ashton P, Iezzi R, et al. Sustained delivery fluocinolone acetonide vitreous implants: long-term benefit in patients with chronic diabetic macular edema. Ophthalmology 2014;121:1892-903. 


\section{Figure Legends}

Figure 1. Mean central subfield thickness (CSFT) in relation to number of days of follow up in eyes treated with $0.3 \mathrm{mg}$ intravitreal ranibizumab for refractory diabetic macular edema.

Figure 2. Mean central subfield thickness (CSFT) in relation to number of $0.3 \mathrm{mg}$ intravitreal ranibizumab injections in eyes treated for refractory diabetic macular edema

Figure 3. Mean logMAR visual acuity (VA) in relation to number of days of follow up in eyes treated with $0.3 \mathrm{mg}$ intravitreal ranibizumab for refractory diabetic macular edema.

Figure 4. Mean logMAR visual acuity (VA) in relation to number of $0.3 \mathrm{mg}$ intravitreal ranibizumab injections in eyes treated for refractory diabetic macular edema 